\title{
LOS CONOCIMIENTOS DE PROPIEDAD INTELECTUAL Y SU INFLUENCIA EN LA RECAUDACIÓN DE DERECHOS DE AUTOR EN LA CIUDAD DE TACNA.
}

\section{INTELLECTUAL PROPERTY KNOWLEDGE AND ITS INFLUENCE ON THE COLLECTION OF COPYRIGHTS IN THE CITY OF TACNA}

\author{
Elam Mizraim Ponce Valencia ${ }^{1}$ \\ ORCID: https://orcid.org/0000-0002-5185-6613 \\ eponcev@cajaarequipa.pe \\ Ricardo Segundo Zapata Ruiz ${ }^{1}$ \\ ORCID: https://orcid.org/0000-0002-5031-2278 \\ riczapatar@virtual.upt.pe
}

\begin{abstract}
RESUMEN
Se realizó un estudio con el objetivo de determinar la influencia de los conocimientos de propiedad intelectual en la recaudación de derechos de autor en la ciudad de Tacna. La metodología empleada es de tipo básica, con un diseño no experimental, transversal y explicativo dirigida a evaluar a una muestra de 162 usuarios de APDAYC, empleando como técnica la encuesta e instrumento el cuestionario. Los resultados muestran un nivel de conocimientos muy alto sobre propiedad intelectual para el pago de derechos de autor (89\%). El nivel de la recaudación de derechos de autor es alto (84\%) de cumplimiento, y que indican además de cumplimiento de los pagos, niveles de morosidad bajos. El trabajo concluye que los conocimientos de propiedad intelectual influyen de forma significativa sobre la recaudación de derechos de autor en la ciudad de Tacna, dada la prueba de correlación de Pearson, que demuestra que existe una relación significativa entre propiedad intelectual y recaudación según el valor de significancia 0.01 y el R-cuadrado que explica que la recaudación de derechos se ve influenciada en un $37.5 \%$ por los conocimientos sobre propiedad intelectual. Finalmente se demostró que la conciencia sobre los pagos de derechos son determinantes sobre el valor recaudado y el cumplimiento de la obligación.
\end{abstract}

Palabra Claves: Conocimientos, Propiedad Intelectual, Recaudación, Derecho de autor.

\begin{abstract}
A study was carried out with the aim of determining the influence of knowledge of intellectual property on the collection of copyright in the city of Tacna. The methodology used is basic, with a non-experimental, cross-sectional and explanatory design aimed at evaluating a sample of 162 users of APDAYC, using the survey technique and the questionnaire as an instrument. The results show a very high level of knowledge of intellectual property for the payment of royalties (89\%). The level of copyright collection compliance is high (84\%), and they indicate low levels of late payment in addition to payment compliance. The paper
\end{abstract}

\footnotetext{
${ }^{1}$ Universidad Privada de Tacna. Facultad de Ciencias Empresariales.
} 
concludes that knowledge of intellectual property has a significant influence on copyright collection in the city of Tacna, given the Pearson correlation test, which shows that there is a significant relationship between intellectual property and collection at the 0.01 significance value, and the R-squared which explains that copyright collection is influenced by knowledge of intellectual property by $37.5 \%$. Finally, it was shown that awareness of royalty payments is a determinant of the value collected and the fulfilment of the obligation.

Keywords: Knowledge, Intellectual Property, Collection, Copyright.

\section{INTRODUCCIÓN}

Un problema que enfrenta el Estado peruano y que puede generar retrasos para el cumplimiento de los objetivos de desarrollo corresponde la recaudación, el cual es uno de los principales objetivos de fuentes de recurso para el Estado peruano.

Entre los otros impuestos, una de las entidades encargadas de recaudación, correspondientes a derechos de autor es APDAYC, la cual estima cobros mensuales que ascienden de 30 a 40 mil soles, con el objetivo que los usuarios cumplan con el derecho del autor para la difusión de música pública. Se estima que de las discotecas y karaokes solo el $95 \%$ cumplen con los pagos correspondientes, de video pubs y bares el $85 \%$, música secundaria, hoteles, restaurantes, gimnasios el $60 \%$, y las tarifas varían de acuerdo a la cantidad de personas que asisten al establecimiento y según horarios. (Correo, 2017)

Estas cifras se replican en la ciudad de Tacna, con un gran número de usuarios que no cumplen con las obligaciones tributarias, afectando la labor de recaudación de la entidad, y que dan una lectura de una propiedad intelectual debilitada, donde es común encontrar casos de elusión y evasión tributaria.

Cabe mencionar que dicha falta de sensibilidad para pagar las tasas y tributos correspondientes a derechos de autor no solo atenta contra la generación de ingresos de los entes reguladores, sino que es una amenaza para los objetivos del estado y los propios autores, dado que no se respeta sus derechos de autoría y se hace un uso inadecuado dado que no existen los permisos requeridos para explotar todo aquello relacionado a materia intelectual. En caso que las entidades reguladoras no logren generar mayor sensibilidad sobre el respeto de los derechos de autor en términos tributarios, los derechos continuarán siendo vulnerados y ello traerá consigo mayor desorden e incumplimiento de la ley que protege a los autores.

Por ello, la presente investigación se enfoca en analizar y estudiar cómo es que el nivel de conocimientos acerca de la propiedad intelectual tiene influencia sobre la recaudación de derechos de autor en la ciudad de Tacna. Por lo que se requiere que se ponga en práctica metodologías científicas, por medio de instrumentación, tales como cuestionarios validados, que además de ser de utilidad para el presente estudio, pueden también servir de marco de referencia para futuras investigaciones. Los resultados que se obtengan permitirían que APDAYC, a partir de ellos, tome decisiones para mejorar sus estrategias de recaudación y de capacitación de sus usuarios respecto a la importancia de contribuir con una adecuada tributación.

Para Molano (2018), en términos de impuesto al valor agregado, las ventas de activos fijos no necesitan pagar impuestos relevantes, y la mayoría de los activos de propiedad industrial son activos fijos de unidades de producción con condiciones de producción fijas, por lo que también se reduce el propósito de cobrar pagos por eventos, es necesario definir los activos intangibles en materia tributaria para que pueda definir claramente la ocurrencia de eventos. Por otro lado, todos los activos intangibles de propiedad intelectual suelen estar gravados, o como máximo, con tasas impositivas diferentes para no obstaculizar las industrias creativas, ciencia o industria de investigación y desarrollo. Arellano (2014), en España. Tuvo como objetivo analizar cuál es la incidencia - si es que existe - de la naturaleza jurídica que posee la propiedad intelectual, en la valoración tributaria que se 
haga de un determinado hecho, sin dejar de considerar las razones económicas que pudieran existir; y concluyó que en contraposición a la escasa relevancia que tiene la naturaleza jurídica de la propiedad intelectual en el diseño normativo de su tributación, sí es relevante al aplicar las normas tributarias que definen su imposición, así pues, la calificación jurídica de un determinado instituto es determinante a la hora de establecer su imposición. En cambio Pérez (2000), muestra que el rol de la política fiscal como regulador de la economía y herramienta de control de los ingresos y gastos nacionales implica un análisis en profundidad de las políticas macroeconómicas globales. Analizar la política fiscal sin tener en cuenta el logro de los objetivos de política financiera, económica o cambiaria. De manera similar, al analizar la política fiscal, no podemos ignorar la dependencia de la política fiscal del nivel económico de un país o de la estructura de producción que representa. Estos son elementos complementarios e intrínsecos en el análisis de correlación y deben ser considerados. García (2017), manifestó que con el desarrollo de la tecnología, es necesario actualizar los métodos y / o comportamientos de protección de derechos de autor, porque ya se han actualizado de otras formas, se han desarrollado leyes (como Brasil y España). El impacto de los derechos de autor de las obras en formato digital es la difusión de obras protegidas en Internet sin la autorización previa por escrito del propietario. Juan de Dios (2017), Dijo que a mayor difusión, mayor efectividad de la ley, y menor difusión, significa que la ley no es válida y la difusión es insuficiente, por lo que la ley es inválida.

De acuerdo con los resultados de la encuesta, Mogollón (2014), dijo que el nivel de derechos de propiedad intelectual de los empresarios de Chiclayo durante 2012 fue muy bajo, lo que muestra que los contribuyentes de Chiclayo no entendieron sus obligaciones tributarias.

Béjar (2017), mostró a nivel regional que el nivel de derechos de propiedad intelectual de los contribuyentes en el nuevo formulario de registro único simplificado en la zona de Gregorio Albarracín Lanchipa es muy bajo, debido a que se observa una gran proporción de la población de investigación $(79,8 \%)$ pagar impuestos a tiempo, lo que indica que los contribuyentes no tienen ningún incentivo para comprender y comprender los problemas fiscales. La estrategia de incentivo a la propiedad intelectual que brinda el Gobierno Provincial de Tacna a los contribuyentes tiene cierto nivel de aplicación convencional y tiene una buena tendencia de desarrollo.

APDAYC: Entidad, en el Perú, cuya misión es proteger los derechos de comunicación pública de nuestros autores, otorgar las licencias de uso de la música y administrar con equidad las regalías recaudadas (APDAYC, 2018).

Derechos de autor: Derechos de los creadores sobre sus obras literarias y artísticas (WIPO, 2019).

Diseño Industrial: Son aspectos ornamentales o estéticos de un objeto (OMPI, 2003).

Impuesto: Tributo que se establece y se pide según sea la capacidad financiera de aquellos que no están exentos de abonarlo (Pérez \& Gardey, 2008).

Marca: Signo distintivo que indica que ciertos productos o servicios han sido realizados o proporcionados por una persona o empresa en particular (OMPI, 2003).

Patente: Derecho exclusivo otorgado sobre una invención: el producto o proceso que constituye una nueva forma de hacer algo, o propone una nueva solución técnica a un problema (OMPI, 2003).

Propiedad intelectual: creaciones de la mente: inventos, obras literarias y artísticas, así como símbolos, nombres e imágenes utilizados en el comercio (OMPI, 2003).

Recaudación: Recoger una suma concreta de dinero. (Pérez \& Merino, 2013)

\section{OBJETIVOS}

Determinar la influencia de los conocimientos de propiedad intelectual en la recaudación de derechos de autor en la ciudad de Tacna.

Evaluar el nivel de conocimientos de propiedad intelectual para el pago de derechos de autor de la ciudad de Tacna y analizar el nivel de la recaudación de derechos de autor en la ciudad de Tacna. 


\section{METODOLOGÍA}

La investigación es de tipo básica no experimental, transversal. Este tipo de investigación se enfoca en realizar una evaluación del contexto problemático permaneciendo sobre las bases teóricas analizadas, a fin de ampliarlas y contrastarlas con la realidad problemática correspondiente a la cultura y recaudación tributaria de APDAYC.

La investigación se ejecuta en la entidad APDAYC, en el periodo 2019, pero tomando en consideración los resultados del ejercicio de recaudación del 2018. La población de estudio está comprendida por 277 usuarios. La muestra se compone por un total de 161 usuarios. Los datos recolectados en función de los instrumentos aplicados serán procesados en el software de corte estadístico IBM SPSS Windows, con el cual se ingresarán los datos de cada encuesta, y se obtendrán las tablas de información, en los cuales se representará los resultados a través de frecuencias porcentuales. Para lograr determinar la influencia se aplicará la prueba de regresión lineal, obteniendo los coeficientes como el R-Cuadrado y el Valor-P, que determinen la influencia y relación entre las variables.

La técnica que se empleará es la encuesta y el instrumento, en función de la técnica que se aplicó fue un cuestionario.

\section{RESULTADOS}

De acuerdo al régimen tributario, los empresarios encuestados mayoritariamente pertenecen al régimen Mype tributario con el $47.8 \%$, correspondiente a un total de 77 empresas.

Tabla 1

Régimen Tributario

\begin{tabular}{llcc}
\hline & & $\mathrm{n}$ & $\%$ \\
\hline & Nuevo RUS & 40 & $24.80 \%$ \\
Régimen Tributario & 44 & $27.30 \%$ \\
& Régimen General & 77 & $47.80 \%$ \\
& Régimen Mype Tributario & 161 & $100.00 \%$ \\
\hline
\end{tabular}

El ítem "El pago de los derechos de propiedad intelectual es parte de una visión responsable del mundo que debe tener los empresarios", permite evaluar el indicador Visión del mundo, correspondiente a la dimensión Conciencia sobre el pago de derechos, para medir la variable Propiedad Intelectual. Los resultados muestran que los empresarios encuestados indican percepciones positivas en sus mayores frecuencias que dan cuenta que consideran que es sumamente importante que se realice el pago de los derechos de propiedad intelectual, el cual debe de formar parte una visión responsable frente al contexto en el que se desenvuelven. Este resultado se debe al $85.1 \%$ de empresarios que indicaron estar totalmente de acuerdo con la afirmación, y el 75\% que indicaron estar de acuerdo con ella.

El ítem "Cumplo con el pago de los derechos de propiedad intelectual a fin de contribuir con responsabilidad con el estado, cumpliendo con mi responsabilidad como ciudadano". Los resultados muestran que los empresarios consideran que se da cumplimiento de los pagos a fin de contribuir de forma responsable con el estado, el cual corresponde a una responsabilidad como ciudadanos. Este resultado se debe al $84.5 \%$ de empresarios que indicaron estar totalmente de acuerdo con la afirmación, y el 10.6\% que indicaron estar de acuerdo con ella.

El ítem "Cuento con valores personales que me hacen responsable para cumplir con el pago de los derechos de propiedad intelectual", permite evaluar el indicador Valores personales. Los resultados muestran percepciones positivas en mayor proporción cuentan con valores personales que los hace responsables para dar cumplimento de pagos de derechos de propiedad intelectual, lo que garantiza, en un plano ético, que no habrá evasión de dicho compromiso. Este resultado se debe al 85.1\% de empresarios que indicaron estar totalmente de acuerdo con la afirmación. 
El ítem "Cuento con la educación cívica suficiente para entender la necesidad de realizar los pagos de derechos de propiedad intelectual", permite evaluar el indicador Valores personales. Los resultados muestran que los empresarios dan cuenta que consideran que poseen la educación cívica necesaria que les invita a considerar que es necesario realizar los pagos de derechos de propiedad intelectual. Es decir, están instruidos sobre su importancia y el deber de cumplir con ello. Este resultado se debe al $85.1 \%$ de empresarios que indicaron estar totalmente de acuerdo con la afirmación, y el 9.9\% que indicaron estar de acuerdo con ella.

Tabla 2

Propiedad intelectual según la dimensión Conciencia sobre el pago de derechos

\begin{tabular}{|c|c|c|c|}
\hline \multicolumn{2}{|c|}{ Dimensión 1: Conciencia sobre el pago de derechos } & \multirow[t]{2}{*}{$\mathbf{n}$} & \multirow[t]{2}{*}{$\%$} \\
\hline & Visión responsable & & \\
\hline \multirow{5}{*}{$\begin{array}{l}\text { El pago de los derechos de propiedad } \\
\text { intelectual es parte de una visión } \\
\text { responsable del mundo que debe tener } \\
\text { los empresarios. }\end{array}$} & Totalmente en desacuerdo & 4 & $2.50 \%$ \\
\hline & Ni de acuerdo ni en desacuerdo & 8 & $5.00 \%$ \\
\hline & De acuerdo & 12 & $7.50 \%$ \\
\hline & Totalmente de acuerdo & 137 & $85.10 \%$ \\
\hline & Total & 161 & $100.00 \%$ \\
\hline \multicolumn{4}{|c|}{ Contribución responsable con el estado } \\
\hline \multirow{4}{*}{$\begin{array}{l}\text { Cumplo con pago de los derechos de } \\
\text { propiedad intelectual cumpliendo con } \\
\text { mi responsabilidad como ciudadano. }\end{array}$} & Ni de acuerdo ni en desacuerdo & 8 & $5.00 \%$ \\
\hline & De acuerdo & 17 & $10.60 \%$ \\
\hline & Totalmente de acuerdo & 136 & $84.50 \%$ \\
\hline & Total & 161 & $100.00 \%$ \\
\hline \multicolumn{4}{|c|}{ Valores personales } \\
\hline \multirow{4}{*}{$\begin{array}{l}\text { Cuento con valores personales que me } \\
\text { hacen responsable para cumplir con el } \\
\text { pago de los derechos de propiedad } \\
\text { intelectual. }\end{array}$} & Ni de acuerdo ni en desacuerdo & 4 & $2.50 \%$ \\
\hline & De acuerdo & 20 & $12.40 \%$ \\
\hline & Totalmente de acuerdo & 137 & $85.10 \%$ \\
\hline & Total & 161 & $100.00 \%$ \\
\hline \multicolumn{4}{|c|}{ Educación cívica } \\
\hline \multirow{4}{*}{$\begin{array}{l}\text { Cuento con la educación cívica } \\
\text { suficiente para entender la necesidad } \\
\text { de realizar los pagos de derechos de } \\
\text { propiedad intelectual. }\end{array}$} & Ni de acuerdo ni en desacuerdo & 8 & $5.00 \%$ \\
\hline & De acuerdo & 16 & $9.90 \%$ \\
\hline & Totalmente de acuerdo & 137 & $85.10 \%$ \\
\hline & Total & 161 & $100.00 \%$ \\
\hline \multicolumn{4}{|c|}{ Normas reguladas } \\
\hline \multirow{5}{*}{$\begin{array}{l}\text { Estoy de acuerdo con los principios y } \\
\text { normas reguladas por el Estado para dar } \\
\text { cumplimiento al pago de los derechos } \\
\text { de propiedad intelectual. }\end{array}$} & Totalmente en desacuerdo & 20 & $12.40 \%$ \\
\hline & Ni de acuerdo ni en desacuerdo & 32 & $19.90 \%$ \\
\hline & De acuerdo & 49 & $30.40 \%$ \\
\hline & Totalmente de acuerdo & 60 & $37.30 \%$ \\
\hline & Total & 161 & $100.00 \%$ \\
\hline \multicolumn{4}{|c|}{ Principios equitativos } \\
\hline & Totalmente en desacuerdo & 20 & $12.40 \%$ \\
\hline Considero que los principios y normas & En desacuerdo & 4 & $2.50 \%$ \\
\hline para el pago de los derechos de & Ni de acuerdo ni en desacuerdo & 45 & $28.00 \%$ \\
\hline \multirow{3}{*}{$\begin{array}{l}\text { propiedad intelectual son equitativas, lo } \\
\text { que alienta mi compromiso. }\end{array}$} & De acuerdo & 52 & $32.30 \%$ \\
\hline & Totalmente de acuerdo & 40 & $24.80 \%$ \\
\hline & Total & 161 & $100.00 \%$ \\
\hline \multicolumn{4}{|c|}{ Programas de pago } \\
\hline \multirow{5}{*}{$\begin{array}{l}\text { Los programas de pago de derechos de } \\
\text { autor me motivan para hacerme } \\
\text { responsable y cumplir con ello. }\end{array}$} & Totalmente en desacuerdo & 20 & $12.40 \%$ \\
\hline & Ni de acuerdo ni en desacuerdo & 8 & $5.00 \%$ \\
\hline & De acuerdo & 77 & $47.80 \%$ \\
\hline & Totalmente de acuerdo & 56 & $34.80 \%$ \\
\hline & Total & 161 & $100.00 \%$ \\
\hline \multicolumn{4}{|c|}{ Motivación de INDECOPI } \\
\hline \multirow{6}{*}{$\begin{array}{l}\text { INDECOPI es una entidad que motiva de } \\
\text { forma adecuada el pago de derechos de } \\
\text { autor. }\end{array}$} & Totalmente en desacuerdo & 29 & $18.00 \%$ \\
\hline & En desacuerdo & 4 & $2.50 \%$ \\
\hline & Ni de acuerdo ni en desacuerdo & 24 & $14.90 \%$ \\
\hline & De acuerdo & 40 & $24.80 \%$ \\
\hline & Totalmente de acuerdo & 64 & $39.80 \%$ \\
\hline & Total & 161 & $100.00 \%$ \\
\hline
\end{tabular}


El ítem "Estoy de acuerdo con los principios y normas reguladas por el Estado para dar cumplimiento al pago de los derechos de propiedad intelectual", permite evaluar el indicador Procesos y normas. Los resultados muestran que los empresarios consideran que están de acuerdo con lo que establece el estado en relación al pago exigido. Este resultado se debe al $37.3 \%$ de empresarios que indicaron estar totalmente de acuerdo con la afirmación, y el 30.4\% que indicaron estar de acuerdo con ella.

El ítem "Considero que los principios y normas para el pago de los derechos de propiedad intelectual son equitativas, lo que alienta mi compromiso", permite evaluar el indicador Procesos y normas. Los resultados muestran que los empresarios consideran que las normativas para el pago de derechos son equitativas, lo cual motiva a que se realice la contribución dado el compromiso. Este resultado se debe al $24.8 \%$ de empresarios que indicaron estar totalmente de acuerdo con la afirmación, y el $32.3 \%$ que indicaron estar de acuerdo con ella.

El ítem "Los programas de pago de derechos de autor me motivan para hacerme responsable y cumplir con ello", permite evaluar el indicador Motivación. Los resultados muestran que los empresarios consideran que se sienten motivados para cumplir con sus obligaciones de pago debido a que existen programas que ofrecen las facilidades para realizarlo. Este resultado se debe al $34.8 \%$ de empresarios que indicaron estar totalmente de acuerdo con la afirmación, y el $47.8 \%$ que indicaron estar de acuerdo con ella.

El ítem "INDECOPI es una entidad que motiva de forma adecuada el pago de derechos de autor", permite evaluar el indicador Motivación. Los resultados muestran que los empresarios encuestados indican percepciones positivas en sus mayores frecuencias que dan cuenta que consideran que INDECOPI como entidad, motiva de forma adecuada a que se realice el pago de derechos de autor.

Este resultado se debe al $39.8 \%$ de empresarios que indicaron estar totalmente de acuerdo con la afirmación, y el $24.8 \%$ que indicaron estar de acuerdo con ella. (Tabla 02)

El ítem "Tengo conocimiento de la Ley de Derechos de Autor y las responsabilidades que demandan", permite evaluar el indicador Norma Vigente, correspondiente a la dimensión Normativas, para medir la variable Propiedad Intelectual. Los resultados muestran que los empresarios encuestados indican percepciones regulares en sus mayores frecuencias que dan cuenta que consideran que los empresarios no tienen el conocimiento debido respecto a la Ley de Derechos de Autor y cuáles son las responsabilidades que esta demanda. Este resultado se debe al $54.7 \%$ de empresarios que indicaron estar ni de acuerdo $n$ en desacuerdo con la afirmación.

El ítem "Cumplo con las normas establecidas en la Ley de Derechos de Autor a fin de respetar los derechos de propiedad intelectual", permite evaluar el indicador Norma Vigente. Los resultados muestran que los empresarios consideran que cumplen con las normas que establece la Ley de Derechos de autor, lo que permite dar respeto de los derechos de propiedad intelectual. Este resultado se debe al $82 \%$ de empresarios que indicaron estar totalmente de acuerdo con la afirmación, y el 13\% que indicaron estar de acuerdo con ella.

El ítem "Los procesos empresariales en los que difundo material de terceros se realiza bajo un estricto cumplimiento de los derechos y tasas que demandan", permite evaluar el indicador Pago de derechos y tasas. Los resultados muestran que los empresarios encuestados indican percepciones positivas en sus mayores frecuencias que dan cuenta que consideran que existe un respeto por el material de terceras personas, la misma que dada su difusión, conlleva un cumplimiento de los derechos y tasas exigibles. Este resultado se debe al $80.1 \%$ de empresarios que indicaron estar totalmente de acuerdo con la afirmación, y el 19.9\% que indicaron estar de acuerdo con ella.

El ítem "Cumplo con pagar las tasas impuestas por uso y/o difusión de material de terceros", permite evaluar el indicador Pago de derechos y tasas. Los resultados muestran que los empresarios consideran que, para la difusión de material de propiedad de terceros, cumplen con el pago de las tasas correspondientes, lo que da a conocer que existe un respeto por el mismo. Este resultado se 
debe al $87.6 \%$ de empresarios que indicaron estar totalmente de acuerdo con la afirmación, y el 5\% que indicaron estar de acuerdo con ella.

Tabla 3

Propiedad intelectual según la dimensión Normativas

\begin{tabular}{|c|c|c|c|}
\hline \multicolumn{2}{|l|}{ Dimensión 2: Normativas } & \multirow[t]{2}{*}{$\mathbf{n}$} & \multirow[t]{2}{*}{$\%$} \\
\hline \multicolumn{2}{|c|}{ Conocimiento de la ley } & & \\
\hline \multirow{6}{*}{$\begin{array}{l}\text { Tengo conocimiento de la Ley de Derechos de Autor } \\
\text { y las responsabilidades que demandan. }\end{array}$} & Totalmente en desacuerdo & 17 & $10.60 \%$ \\
\hline & En desacuerdo & 4 & $2.50 \%$ \\
\hline & Ni de acuerdo ni en desacuerdo & 88 & $54.70 \%$ \\
\hline & De acuerdo & 36 & $22.40 \%$ \\
\hline & Totalmente de acuerdo & 16 & $9.90 \%$ \\
\hline & Total & 161 & $100.00 \%$ \\
\hline \multicolumn{4}{|c|}{ Cumplimiento de normas según ley } \\
\hline \multirow{4}{*}{$\begin{array}{l}\text { Cumplo con las normas establecidas en la Ley de } \\
\text { Derechos de Autor a fin de respetar los derechos de } \\
\text { propiedad intelectual. }\end{array}$} & Ni de acuerdo ni en desacuerdo & 8 & $5.00 \%$ \\
\hline & De acuerdo & 21 & $13.00 \%$ \\
\hline & Totalmente de acuerdo & 132 & $82.00 \%$ \\
\hline & Total & 161 & $100.00 \%$ \\
\hline \multicolumn{4}{|c|}{ Difusión de material de terceros } \\
\hline \multirow{3}{*}{$\begin{array}{l}\text { Los procesos empresariales en los que difundo } \\
\text { material de terceros se realiza en estricto } \\
\text { cumplimiento de derechos y tasas }\end{array}$} & De acuerdo & 32 & $19.90 \%$ \\
\hline & Totalmente de acuerdo & 129 & $80.10 \%$ \\
\hline & Total & 161 & $100.00 \%$ \\
\hline \multicolumn{4}{|c|}{ Pago de tasas e impuestos } \\
\hline \multirow{4}{*}{$\begin{array}{l}\text { Cumplo con pagar las tasas impuestas por uso y/o } \\
\text { difusión de material de terceros. }\end{array}$} & Ni de acuerdo ni en desacuerdo & 12 & $7.50 \%$ \\
\hline & De acuerdo & 8 & $5.00 \%$ \\
\hline & Totalmente de acuerdo & 141 & $87.60 \%$ \\
\hline & Total & 161 & $100.00 \%$ \\
\hline \multicolumn{4}{|c|}{ Operaciones sujetas a pagos } \\
\hline \multirow{3}{*}{$\begin{array}{l}\text { Las operaciones que realizo en la empresa están } \\
\text { sujetas a pago de impuestos y cumplimiento de } \\
\text { pago de tasas. }\end{array}$} & De acuerdo & 4 & $2.50 \%$ \\
\hline & Totalmente de acuerdo & 157 & $97.50 \%$ \\
\hline & Total & 161 & $100.00 \%$ \\
\hline \multicolumn{4}{|c|}{ Pago como impedimento } \\
\hline \multirow{3}{*}{$\begin{array}{l}\text { El pago de impuestos no son un impedimento para } \\
\text { que cumpla con los derechos de propiedad } \\
\text { intelectual. }\end{array}$} & De acuerdo & 12 & $7.50 \%$ \\
\hline & Totalmente de acuerdo & 149 & $92.50 \%$ \\
\hline & Total & 161 & $100.00 \%$ \\
\hline \multicolumn{4}{|c|}{ Inferencia de derechos y tasas en los precios de mercado } \\
\hline \multirow{3}{*}{$\begin{array}{l}\text { El cumplimiento de los derechos y tasas por } \\
\text { derechos de propiedad intelectual no debe de } \\
\text { inferir en la oferta de precios al mercado. }\end{array}$} & De acuerdo & 17 & $10.60 \%$ \\
\hline & Totalmente de acuerdo & 144 & $89.40 \%$ \\
\hline & Total & 161 & $100.00 \%$ \\
\hline \multicolumn{4}{|c|}{ Disminución de ventas } \\
\hline \multirow{3}{*}{$\begin{array}{l}\text { Está de acuerdo en que al considerar el Impuesto } \\
\text { Selectivo al Consumo en los precio de sus } \\
\text { productos, disminuiría sus ventas. }\end{array}$} & Totalmente en desacuerdo & 4 & $2.50 \%$ \\
\hline & Totalmente de acuerdo & 157 & $97.50 \%$ \\
\hline & Total & 161 & $100.00 \%$ \\
\hline
\end{tabular}

El ítem "Las operaciones que realizo en la empresa están sujetas a pago de impuestos y cumplimiento de pago de tasas", permite evaluar el indicador Pago de derechos y tasas. Los resultados muestran que los empresarios consideran que las actividades que desarrollan a nivel operativo consideran como una responsabilidad cumplir con el pago de las tasas correspondientes a la propiedad intelectual, es decir, que esta forma parte de su lista de responsabilidades. Este resultado se debe al 97.5\% de empresarios que indicaron estar totalmente de acuerdo con la afirmación, y el $2.5 \%$ que indicaron estar de acuerdo con ella.

El ítem "El pago de impuestos no son un impedimento para que cumpla con los derechos de propiedad intelectual", los resultados muestran que los empresarios consideran que las imposiciones establecidas para los derechos de propiedad intelectual no son impedimento para que este se ejecute. Es decir, existe cumplimiento sin alguna cuestión que restrinja esta responsabilidad. Este resultado se 
debe al 92.5\% de empresarios que indicaron estar totalmente de acuerdo con la afirmación, y el 7.5\% que indicaron estar de acuerdo con ella.

El ítem "El cumplimiento de los derechos y tasas por derechos de propiedad intelectual no debe de inferir en la oferta de precios al mercado". Los resultados muestran que los empresarios consideran que el cumplimiento de los derechos correspondientes al pago no es causal para que decidan realizar cambio en los precios de mercado de los bienes o servicios que ofertan, es decir, que no es un condicionante de los mismos. Este resultado se debe al $89.4 \%$ de empresarios que indicaron estar totalmente de acuerdo con la afirmación, y el 10.6\% que indicaron estar de acuerdo con ella.

El ítem "Está de acuerdo en que, al considerar el Impuesto Selectivo al Consumo en los precios de sus productos, disminuiría sus ventas". Los resultados muestran que los empresarios consideran que la aplicación de un impuesto selectivo al consumo sobre sus precios si es un factor que puede generar disminución sobre las ventas, bajo la consideración que ello puede encarecer el precio ofertado. Este resultado se debe al $\mathbf{9 7 . 5 \%}$ de empresarios que indicaron estar totalmente de acuerdo con la afirmación. (Tabla 3)

\section{Tabla 4}

Recaudación

\begin{tabular}{|c|c|c|c|c|}
\hline & RECAUDACIÓN & & $\mathbf{n}$ & $\%$ \\
\hline \multirow{7}{*}{$\begin{array}{l}\text { Valor } \\
\text { recaudado }\end{array}$} & \multicolumn{4}{|c|}{ Valor de pago } \\
\hline & \multirow{6}{*}{$\begin{array}{l}\text { Indique el valor de pago de } \\
\text { derechos (en S/.) según } \\
\text { cumplimiento de pago de } \\
\text { derechos de propiedad } \\
\text { intelectual. (Mensual) }\end{array}$} & Menos de S/.100 & 124 & $77.00 \%$ \\
\hline & & Entre $S / .100$ y $S / .200$ & 21 & $13.00 \%$ \\
\hline & & Entre $S / .200$ y $S / .300$ & 4 & $2.50 \%$ \\
\hline & & Entre $S / .300$ y $S / .400$ & 0 & $0.00 \%$ \\
\hline & & Más de $S / .400$ & 12 & $7.50 \%$ \\
\hline & & Total & 161 & $100.00 \%$ \\
\hline \multirow{12}{*}{$\begin{array}{c}\text { Cumplimiento de las } \\
\text { obligaciones }\end{array}$} & \multicolumn{4}{|c|}{ Nivel de cumplimiento de pagos } \\
\hline & \multirow{5}{*}{$\begin{array}{l}\text { Indique el nivel de cumplimiento } \\
\text { del pago de derechos de } \\
\text { propiedad intelectual }\end{array}$} & Bajo & 8 & $5.00 \%$ \\
\hline & & Regular & 29 & $18.00 \%$ \\
\hline & & Alto & 48 & $29.80 \%$ \\
\hline & & Muy alto & 76 & $47.20 \%$ \\
\hline & & Total & 161 & $100.00 \%$ \\
\hline & \multicolumn{4}{|c|}{ Grado de morosidad } \\
\hline & \multirow{5}{*}{$\begin{array}{l}\text { Indique el grado de morosidad } \\
\text { para pagar los derechos de } \\
\text { propiedad intelectual }\end{array}$} & Alto & 8 & $5.00 \%$ \\
\hline & & Regular & 29 & $18.00 \%$ \\
\hline & & Bajo & 48 & $29.80 \%$ \\
\hline & & Muy bajo & 76 & $47.20 \%$ \\
\hline & & Total & 161 & $100.00 \%$ \\
\hline
\end{tabular}

El ítem "Indique el valor de pago de derechos (en S/.) según cumplimiento de pago de derechos de propiedad intelectual. (Mensual)", permite evaluar el indicador Valor correspondiente a la dimensión Valor recaudado, para medir la variable Recaudación.

Los resultados muestran que los empresarios encuestados indican que la contribución que realizan, en sus mayores frecuencias, es menor de $\mathrm{S} / .100 .00$, correspondiente a un $77 \%$, seguido por el $13 \%$ que realiza aportes de entre $S / .100 .00$ a $S / .200 .00$.

Por otro lado, la menor frecuencia corresponde al $2.5 \%$ que indicaron aportar entre $S / .200$ y S/.300.00.

El ítem "Indique el nivel de cumplimiento del pago de derechos de propiedad intelectual", permite evaluar el indicador Nivel de cumplimiento del pago, correspondiente a la dimensión Cumplimiento de las obligaciones, para medir la variable Recaudación.

Los resultados muestran que los empresarios encuestados indican percepciones positivas en sus mayores frecuencias que dan cuenta que consideran que tienen un alto grado de cumplimiento de los derechos de propiedad intelectual, es decir que respetan las exigencias que conllevan tales. 
Este resultado se debe al $47.2 \%$ de empresarios que indicaron un muy alto grado de cumplimiento, seguido por el $29.8 \%$ que indicaron un alto grado.

Por otro lado, la menor frecuencia corresponde al $5 \%$ que indicaron cumplir, pero en bajo grado con el pago de derechos de propiedad intelectual.

El ítem "Indique el grado de morosidad para pagar los derechos de propiedad intelectual", permite evaluar el indicador Nivel de morosidad, correspondiente a la dimensión Cumplimiento de las obligaciones, para medir la variable Recaudación.

Los resultados muestran que los empresarios encuestados indican percepciones positivas en sus mayores frecuencias que dan cuenta que consideran que el nivel de morosidad es bajo lo que es un indicador positivo para deducir que existe un pago oportuno de las tasas por derechos de uso de propiedad de terceros.

Este resultado se debe al $47.2 \%$ de empresarios que indicaron tener un muy bajo grado de morosidad para realizar el pago, seguido por el $29.8 \%$ que indicaron tener un bajo grado.

Por otro lado, la menor frecuencia corresponde al $5 \%$ que indicaron presentar un alto grado de morosidad. (tabla 4)

Tabla 5

Evaluación general de la Propiedad Intelectual

\begin{tabular}{|c|c|c|c|c|c|}
\hline Variable & Dimensión & Indicador & Media & Porcentaje & Valoración \\
\hline \multirow{9}{*}{$\begin{array}{l}\text { Propiedad } \\
\text { Intelectual }\end{array}$} & \multirow{4}{*}{$\begin{array}{c}\text { Conciencia sobre el } \\
\text { pago de derechos }\end{array}$} & $\begin{array}{c}\text { Conciencia sobre el pago de } \\
\text { derechos }\end{array}$ & 4.76 & $95 \%$ & Muy alto \\
\hline & & Valores personales & 4.81 & $96 \%$ & Muy alto \\
\hline & & Procesos y normas & 3.67 & $73 \%$ & Alto \\
\hline & & Motivación & 3.79 & $76 \%$ & Alto \\
\hline & \multicolumn{2}{|c|}{ Media de Conciencia sobre el pago de derechos } & 4.26 & $85 \%$ & Muy alto \\
\hline & \multirow{4}{*}{ Normativas } & Normativa vigente & 3.98 & $80 \%$ & Alto \\
\hline & & Pago de derechos y tasas & 4.88 & $98 \%$ & Muy alto \\
\hline & & Media de Normativas & 4.66 & $93 \%$ & Muy alto \\
\hline & & Media de Propiedad Intelectual & 4.46 & $89 \%$ & Muy alto \\
\hline \multirow[t]{3}{*}{ Recaudación } & \multirow{3}{*}{$\begin{array}{l}\text { Cumplimiento de las } \\
\text { obligaciones }\end{array}$} & $\begin{array}{l}\text { Nivel de cumplimiento del pago } \\
\text { correspondiente }\end{array}$ & 4.19 & $84 \%$ & Alto \\
\hline & & Nivel de morosidad & 4.19 & $84 \%$ & Alto \\
\hline & & Media de Recaudación & 4.19 & $84 \%$ & Alto \\
\hline
\end{tabular}

Ajuste del modelo de análisis:

Se propuso medir si los conocimientos de propiedad intelectual influyen de forma significativa en la recaudación de derechos de autor en la ciudad de Tacna.

Tabla 6

Resumen del modelo

\begin{tabular}{llllr}
\hline Modelo & $\mathrm{R}$ & $\mathrm{R}$ cuadrado & R cuadrado corregida & Error típ. de la estimación \\
\hline 1 & $.612(\mathrm{a})$ & .375 & .371 & .71789 \\
\hline a. & Variables predictoras: (Constante), Propiedad Intelectual & &
\end{tabular}

Tabla 7

ANOVA del modelo

\begin{tabular}{llrrrrc}
\hline Modelo & & Suma de cuadrados & gl & Media cuadrática & F & Sig. \\
\hline 1 & Regresión & 49.089 & 1 & 49.089 & 95.251 & $.000(\mathrm{a})$ \\
& Residual & 81.942 & 159 & .515 & & \\
& Total & 131.031 & 160 & & & \\
\hline
\end{tabular}

a. Variables predictoras: (Constante), Propiedad Intelectual

b. Variable dependiente: Recaudación 
Tabla 8

Coeficientes del Modelo

\begin{tabular}{|c|c|c|c|c|c|c|}
\hline \multirow{2}{*}{\multicolumn{2}{|c|}{ Modelo }} & \multicolumn{2}{|c|}{$\begin{array}{l}\text { Coeficientes no } \\
\text { estandarizados }\end{array}$} & \multirow{2}{*}{$\begin{array}{c}\text { Coeficientes } \\
\text { estandarizados }\end{array}$} & \multirow{2}{*}{$\frac{t}{B}$} & \multirow{2}{*}{$\frac{\text { Sig. }}{\text { Error típ. }}$} \\
\hline & & B & Error típ. & & & \\
\hline \multirow[t]{2}{*}{1} & (Constante) & -.740 & .509 & & -1.454 & .148 \\
\hline & Propiedad Intelectual & 1.106 & .113 & .612 & 9.760 & .000 \\
\hline
\end{tabular}

Como se aprecia (Tabla 6,7 y 8 ) el valor de significancia calculado es 0.01 lo que es indicador que existe una relación significativa entre el conocimiento de la propiedad intelectual y la recaudación. Así mismo, el R-cuadrado indica que existe una influencia del $37.5 \%$ del conocimiento de la propiedad intelectual sobre la recaudación.

\section{DISCUSIÓN}

Los resultados dan cuenta que el grado de conocimiento sobre propiedad intelectual por parte de los empresarios evaluados a nivel de cultura para el debido cumplimiento es alta, con una media de 4.46 , es decir, un $89 \%$ de cumplimiento de niveles de conocimiento, lo que determina valores muy altos, y que dan cuenta que existe conciencia sobre el pago de derechos y sobre las normativas en niveles óptimos.

Estos resultados permiten ser fuente de garantía para la recaudación, la misma que también presenta niveles altos, con un $84 \%$ de cumplimiento, y que indica a su vez que existe un nivel de pago correspondiente alto y niveles de morosidad bajos.

Cabe indicar que el cumplimiento de pago de los derechos de propiedad intelectual no es un factor que desmotiva a los empresarios, limitando sus operaciones, y ello se contrasta con los hallazgos de Molano (2018), quien, en su investigación en Colombia, también permitió determinar que el pago de los derechos es un factor que incentiva a la industria.

Crear una cultura de pago de derechos de propiedad intelectual debe de formar parte de los buenos hábitos de los empresarios nacionales, y dada la evaluación existe sensibilización sobre las obligaciones y respeto de los mismos. Estos hallazgos guardan similitud con Arellano (2014), quien determina que ello es un aspecto de corte jurídico aplicable a realidades de diferentes naciones, tales como en España y Chile según su estudio comparado.

Como indica Pérez (2000), y al igual que lo que se muestra con la investigación, el pago de derechos de propiedad intelectual es una herramienta que permite fortalecer la política fiscal del país, debido a que permite que se generen mayores ingresos para el estado.

El pago de derechos de autor está regulado en el derecho peruano, y ello aplica para los diferentes rubros, es por ello que la investigación presente tomó como juicio evaluar a los diferentes contribuyentes sin exclusión del rubro o campo empresarial. Ello es similar a lo que manifiesta García (2017), quien realizó su investigación en un campo de obras de formato digital, o similar al caso de los comerciantes evaluados por Mogollón (2014).

Según nuestros resultados, los conocimientos de propiedad intelectual influyen de forma significativa sobre la recaudación de derechos de autor en la ciudad de Tacna, que explica que la recaudación de derechos se ve influenciada en un $37.5 \%$ por los conocimientos sobre propiedad intelectual. Es decir que la conciencia sobre los pagos de derechos y normativas son determinantes sobre el valor recaudado y el cumplimiento de las obligaciones de los empresarios. A su vez existe un porcentaje de usuarios que cuentan con un nivel de morosidad bajo, pero a considerar, este porcentaje se da debido a que no confían en su totalidad en la sociedad de gestión colectiva y en los fines de lo recaudado, esta situación puede llegar a generar incertidumbre entre los empresarios que cumplen responsablemente con sus pagos y perjudicar los intereses de los autores y compositores. INDECOPI, por medio de la Gerencia de Promoción y Difusión debe de realizar informes públicos para 
que la población tenga conocimiento de los valores recaudados por pago de derechos de propiedad intelectual y el destino de los mismos, promoviendo la transparencia y participación ciudadana a fin que estos mismos puedan fiscalizar el correcto uso de la recaudación.

Evaluar el nivel de conocimientos de propiedad intelectual para el pago de derechos de autor de la ciudad de Tacna es muy alto, dada una media de 4.46 puntos que indica un $89 \%$ de conocimientos sobre el mismo, y que es indicador que los empresarios evaluados poseen niveles de conciencia sobre el pago muy altos lo que indica conciencia, valores, conocimiento de procesos y normas y motivación, como también conocimientos adecuados sobre la normativa vigente y pago de derechos y tasas. Los empresarios actualmente no cuentan con capaciones que abarquen mayor conocimiento en cuestión de derechos de autor, esto puede influir de forma negativa a que los usuarios tomen acciones incorrectas, por desconocimiento, perjudicando así la recaudación. INDECOPI, por medio de su Gerencia Regional en coordinación con la Gerencia de Promoción y Difusión, debe de realizar campañas de información en diferentes instituciones privadas respecto a las obligaciones de todo ciudadano de respetar la propiedad intelectual, y de dar un debido uso en caso que requieran realizar alguna reproducción o difusión del mismo. Este tipo de campañas deben realizarse mediante visitas directas a locales comerciales que ofertan productos y/o servicios sin distinción de la naturaleza del negocio, y deben estar dirigidos principalmente a los dueños, gerentes o administradores de estos.

El nivel de la recaudación de derechos de autor en la ciudad de Tacna es alto, dada una media de 4.19 puntos, es decir un $84 \%$ de cumplimiento, y que indican además de cumplimiento de los pagos, niveles de morosidad bajos. Ello resulta de suma importancia dado que refleja que los empresarios muestran responsabilidad para asumir los compromisos de pago de derechos. Existe un porcentaje menor de morosidad que puede verse influenciado, debido a que los empresarios no cuentan con programas eficientes para gestionar facilidades de pago, y que las entidades competentes no incentivan de forma correcta a los empresarios morosos a cumplir con sus obligaciones pendientes. Por medio de la Gerencia Regional en coordinación con la Gerencia de Supervisión y Fiscalización, se debe de promover programas de descuentos y facilidades de pago a los usuarios que realicen aportes con prontitud y según los plazos establecidos. Ello permitirá generar mayor confianza y compromiso en cuanto al pago de derechos de propiedad intelectual.

Los conocimientos de propiedad intelectual se relacionan de forma significativa con la recaudación de derechos de autor en la ciudad de Tacna, dada la prueba de correlación de Pearson, que demuestra según el valor de significancia 0.01 existe una relación significativa entre propiedad intelectual y recaudación, y que sugiere que la recaudación a nivel de cumplimiento de los pagos y baja morosidad están relacionados con el conocimiento que pueden poseer los empresarios evaluados, los cuales asociación su cumplimiento a tal nivel de conocimiento. Los empresarios morosos en su mayoría no cuentan con una instrucción temprana debida como la educación, valores cívicos, sensibilización, entre otros, este es un factor negativo para la sociedad de gestión colectiva, ya que la conciencia de los usuarios influye en la toma de sus decisiones, ya sea esta acertada o errada, como el pago de sus obligaciones. INDECOPI, por medio de la Gerencia de Promoción y Difusión y a requerimiento de la Gerencia Regional, debe de realizar programas de capacitación en entidades escolares a nivel primario y secundario en los que se promuevan valores cívicos sobre el respeto de la propiedad intelectual y al mismo tiempo se genere conciencia tributaria responsable como contribución con la sociedad y el estado.

\section{REFERENCIAS}

APDAYC. (2018). APDAYC. Obtenido de APDAYC Web Site: http://www.apdayc.org.pe/index.php/nosotros/quienes-somos

APDAYC. (2019). Informe de recaudación anual APDAYC Tacna. Tacna: APDAYC.

APDAYC. (2020). Asociación Peruana de Autores y Compositores. Obtenido de http://www.apdayc.org.pe/ 
Arellano, M. (2014). La propiedad intelectual en el derecho tributario chileno y español. un estudio comparado. España: Universidad de Salamanca.

Béjar, D. (2017). Estrategias tributarias y su influencia en el nivel de recaudación del impuesto predial en la municipalidad provincial de Tacna, año 2015. Tacna: Universidad Nacional Jorge Basadre Grohmann.

Chacaltana, E. (2016). La cultura tributaria de los contribuyentes del nuevo Registro Único Simplificado y su relación con la evasión tributaria en el distrito Gregorio Albarracín Lanchipa periodo 2013. Tacna: Universidad Nacional Jorge Basadre Grohmann.

Congreso de la República. (2003). Ley sobre el Derecho de Autor - DECRETO LEGISLATIVO № 822. Perú: Poder Ejecutivo.

Correo. (10 de Enero de 2017). APDAYC recauda de 30 a 40 mil soles mensualmente en cobros sobre el derecho del autor. Diario El Correo.

García, M. (2017). La protección penal del derecho de autor en relación a la difusión de obras en formato digital en lima 2012 - 2017. Lima: Universidad César Vallejo.

INDECOPI. (2019). https://www.abogadosempresariales.pe. Obtenido de https://www.abogadosempresariales.pe/la-propiedad-intelectual-en-el-peru/

Juan de Dios, V. (2017). La Difusión de La Ley de Propiedad Intelectual y su Eficacia en Huánuco. Huánuco: Universidad de Huánuco.

Mogollón, V. (2014). Nivel De Cultura Tributaria En Los Comerciantes De La Ciudad De Chiclayo En El Periodo 2012 Para Mejorar La Recaudación Pasiva De La Región Chiclayo, Perú. Chiclayo: Universidad Católica Santo Toribio De Mogrovejo.

Molano, D. (2018). Tratamiento tributario de los activos intangibles de propiedad intelectual en el nuevo hecho generador del impuesto al valor agregado (IVA) . Bogotá, Colombia: Universidad Externado de Colombia .

OMPI. (2003). Organización Mundial de la Propiedad Intelectual. Suiza: OMPI N 450(S) ISBN 978-92805-1157-4.

Pérez, J., \& Gardey, A. (2008). Definición. Obtenido de Definición Web Site: https://definicion.de/impuesto/

Pérez, J., \& Gardey, A. (2010). Definición. Obtenido de Definición Web Site: https://definicion.de/tributo/

Pérez, J., \& Merino, M. (2013). Definición. Obtenido de Definición Web Site: https://definicion.de/recaudacion/

Pérez, M. (2000). El sistema tributario en materia de propiedad intelectual: una propuesta de fortalecimiento de la politica fiscal en el Ecuador. Quito, Ecuador: Universidad Andina Simón Bolivar.

Perret, E. (2016). El secreto de la Motivación. Mexico. SquareSpace.

Superintendencia Nacional de Aduanas y de Administración Tributaria. (2012). Cultura Tributaria. Lima: SUNAT.

Tylor, E. (1995). "La ciencia de la cultura". Barcelona, España: Anagrama.

WIPO. (2019). Wipo. Obtenido de Wipo Web Site: https://www.wipo.int/copyright/es/

Yañez, J. (5 de Noviembre de 2010). Evasión versus Elusión / Diario Estrategia. Obtenido de Universidad de Chile Web Site: http://econ.uchile.cl/es/opinion/evasi-n-versus-elusi-n-diarioestrategia 